\title{
Accompagner le développement pédagogique des enseignants universitaires à l'aide d'un cadre conceptuel original
}

Backing up the development of teaching skills of college teachers with an original conceptual frame

Acompañar el desarrollo pedagógico de los catedráticos gracias a un marco conceptual original

Die pädagogische Entwicklung der Dozenten mit einem originalen konzeptuellen Rahmen begleiten

Mariane Frenay, Alenoush Saroyan, K. Lynn Taylor, Denis Bédard, Mieke Clement, Nicole Rege Colet, Jean-Jacques Paul et Anette Kolmos

\section{(2) OpenEdition}

Journals

Édition électronique

URL : http://journals.openedition.org/rfp/2253

DOI : $10.4000 /$ rfp. 2253

ISSN : 2105-2913

Éditeur

ENS Éditions

Édition imprimée

Date de publication : 15 juillet 2010

Pagination : 63-76

ISBN : 978-2-7342-1188-4

ISSN : 0556-7807

\section{Référence électronique}

Mariane Frenay, Alenoush Saroyan, K. Lynn Taylor, Denis Bédard, Mieke Clement, Nicole Rege Colet, Jean-Jacques Paul et Anette Kolmos, « Accompagner le développement pédagogique des enseignants universitaires à l'aide d'un cadre conceptuel original », Revue française de pédagogie [En ligne], 172 I juillet-septembre 2010, mis en ligne le 01 décembre 2014, consulté le 19 avril 2019. URL http://journals.openedition.org/rfp/2253 ; DOI : 10.4000/rfp.2253 


\section{Accompagner le développement pédagogique des enseignants universitaires à l'aide d'un cadre conceptuel original}

\section{Mariane Frenay, Alenoush Saroyan, K. Lynn Taylor, Denis Bédard, Mieke Clement, Nicole Rege Colet, Jean-Jacques Paul et Anette Kolmos}

L'objectif de cet article est de présenter un cadre conceptuel issu d'une expertise collective, développée pendant trois années au sein d'un projet international. Ce cadre permet de formaliser le conseil et l'accompagnement pédagogique au niveau universitaire, en questionnant les défis et enjeux de la pratique du développement pédagogique vis-à-vis des enseignants, des équipes d'enseignants et des responsables des institutions universitaires. Il permet également de soulever un ensemble de pistes de recherches qui restent à développer autour de la thématique du soutien à l'enseignement universitaire.

Descripteurs (TESE) : formation continue des enseignants, évaluation des enseignants, qualité de l'enseignement, promotion de la mobilité, université, politique en matière d'éducation.

\section{LE PROJET « FACDEV » : INITIALEMENT UN PROGRAMME DE FORMATION}

Partant du lancement en 2002 du Réseau européen de recherche et d'innovation pédagogique dans l'enseignement supérieur (RERIES), branche européenne du Global university network on innovation (GUNI) de I'UNESCO, les auteurs de cet article ont soumis un projet de coopération entre l'Union européenne et le Canada, intitulé «FACDEV program: Promoting faculty development to enhance the quality of learning in higher education", afin d'encourager la mobilité entre étudiants et enseignants d'établissements d'enseignement supérieur. Ce projet visait à promouvoir notamment les pratiques de conseil et d'accompagnement pédagogique permettant de maintenir une certaine qualité de l'enseignement universitaire, ainsi qu'à développer un programme de formation pour les personnes souhaitant se spécialiser dans ce domaine.

De cette expérience, qui s'est déroulée de 2002 à 2005, nous retenons principalement les effets directs suivants: le développement et l'implémentation d'un programme international de formation, construit par un processus collaboratif de travail entre sept institutions (Frenay, Saroyan, Clement et al., 2005) et qui a permis à des étudiants (ils étaient plus de 40 à étudier à l'étranger pendant un mois) et à des enseignants de participer à un programme de mobilité centré spécifiquement sur le conseil et l'accompagnement du développement pédagogique des enseignants universitaires. Ces échanges ont permis, par l'intermédiaire des étudiants en mobilité, de proposer au sein des institutions d'accueil une activité 
de formation sur la manière dont se pratique le développement pédagogique au sein de l'institution d'origine et, au retour, d'échanger sur l'expérience vécue. L'objectif étant, à travers ces deux activités de développement pédagogique, de favoriser le partage d'expertise et d'amener les étudiants à mettre en pratique une forme de développement pédagogique différente selon le contexte. Les enseignants en mobilité participaient à ces activités de développement pédagogique de leur institution d'accueil en partageant leurs pratiques de conseil et d'accompagnement et, à leur retour, en suscitant un échange avec leurs collègues, notamment sur les implications possibles pour leur institution d'origine. Ce fut ainsi, pour les Européens, la possibilité d'être confrontés à des pratiques de pédagogie universitaire et d'accompagnement pédagogique solidement ancrées dans les structures institutionnelles. Pour les partenaires canadiens, ce fut une opportunité unique d'être impliqués dans les questions et développements issus du processus de Bologne: ils contribuèrent ainsi notamment à la réflexion sur la manière de concevoir le soutien et l'accompagnement pédagogique des enseignants, des équipes de programmes et des institutions, en prise avec une réforme de programmes sans précédent.

En effet le conseil et l'accompagnement pédagogique, tels qu'ils sont pratiqués et mis en œuvre tant en Europe qu'au Canada, traduisent une histoire et une pratique différentes. Au Canada, c'est à partir de l'insatisfaction des étudiants quant à la qualité de la formation durant les années soixante que se sont créés les premiers services de pédagogie universitaire au sein des universités. Même s'il a fallu du temps pour que chaque institution investisse dans ces services, presque toutes les 93 institutions canadiennes actuelles soutiennent de manière formelle leurs enseignants, pour améliorer la qualité de leur enseignement et l'apprentissage de leurs étudiants. En Europe, certains pays, comme les pays scandinaves et le Royaume-Uni, ont développé la panoplie complète des activités de développement pédagogique, tandis que d'autres, comme la France, n'ont pas ou que très peu développé de telles activités de développement. Cependant, si le projet de recherche que nous avons mené a permis effectivement le développement de ce programme de formation et la participation d'enseignants et d'étudiants, certaines difficultés spécifiques, inhérentes à la réalisation de tels projets internationaux, se sont manifestées, notamment la difficulté d'institutionnaliser par la suite le programme de formation au sein des programmes des institutions partenaires.
Les principaux effets indirects de ce projet ont été la constitution d'un espace collaboratif de recherches et de pratiques spécifiques au conseil et à l'accompagnement du développement pédagogique, espace toujours fonctionnel aujourd'hui. L'observation, le partage et l'analyse des perspectives institutionnelles et nationales, en matière de développement pédagogique, se sont toujours réalisés dans cette articulation entre des pratiques spécifiques et des cadres de référence ancrés sur nos recherches respectives, ce dont chaque pays a ensuite bénéficié en retour. Cette expérience partagée a ainsi démontré l'intérêt de la synergie issue de l'interaction entre les contextes que Rowland qualifie de contextes privés (renvoyant aux pratiques locales), publics (théories partagées) et partagés (débats critiques, voir Rowland, 1999). Des publications et des recherches conjointes se poursuivent. Dans l'une de ces publications, nous avons ainsi produit une analyse approfondie de la manière dont, dans chacun des pays, les services de pédagogie universitaire ou de soutien à l'enseignement universitaire se sont développés et pratiquent aujourd'hui le conseil et l'accompagnement (Saroyan \& Frenay, 2010). Nous y développons une comparaison de cinq études de cas nationales (1), ce qui permet de pointer les similitudes et les différences, et surtout les défis communs et spécifiques posés au développement pédagogique aujourd'hui (Frenay \& Saroyan, 2010). Cette comparaison permet également de discuter de l'influence des politiques institutionnelles et nationales dans la manière dont elles structurent les services de pédagogie universitaire et leurs activités, notamment au travers de l'influence d'organismes de régulation de nature diverse (réseaux de chercheurs, associations professionnelles, groupes de pression, réseaux éducatifs, etc.).

Quand nous avons démarré nos premières activités, nous avons fait le constat qu'il n'existait pas de cadre de référence communément accepté ayant élaboré les concepts spécifiques au développement pédagogique. Au travers de nos échanges, nous nous référions à des concepts différents alors même que nous utilisions les mêmes termes. Partant de ce constat qui, s'il était vrai avec huit partenaires, devait l'être d'autant plus avec tous les partenaires internationaux, nous avons travaillé à la construction d'un cadre conceptuel qui puisse être pertinent dans nos différents contextes et être ainsi partagé, tant pour les pratiques que pour la recherche (2). L'essentiel de cet article explicite ce cadre conceptuel que nous souhaitons partager avec les lecteurs de ce dossier. Le produit fini, présenté ici, a été l'objet de nombreux allers-retours, partant de discussions en petits 
groupes, passant par les premières synthèses et faisant ensuite l'objet de tests auprès de différents publics qui pratiquent tous le développement pédagogique dans leur métier de praticiens ou de chercheurs (Bédard, Clement \& Taylor, 2010). Les cinq dimensions qui constituent ce cadre conceptuel et qui seront décrites dans la suite ont cherché à saisir la diversité des perspectives échangées lors des validations successives. Ce cadre reflète non seulement nos propres analyses et réflexions, mais également la synthèse des écrits réalisée à cette occasion.

\section{UN CADRE CONCEPTUEL POUR FORMALISER ET ÉVALUER LES PRATIQUES DE SOUTIEN AU DÉVELOPPEMENT PÉDAGOGIQUE DES ENSEIGNANTS}

Tout au long de ces années, nous avons pu construire et finaliser un cadre de référence qui nous permet de conceptualiser la pratique et la recherche en développement pédagogique (Taylor \& Rege Colet, 2010). Ce cadre, que nous avons construit collectivement, a été soumis à la critique de professionnels du développement pédagogique, tant de milieux anglophones que francophones (Bédard, Clement \& Taylor, 2010), et il peut aujourd'hui servir différents objectifs, notamment ceux de consolider la formation initiale et continue des conseillers pédagogiques, de piloter et d'évaluer l'efficacité de services de pédagogie universitaire, ou encore de pointer les pistes de recherches à poursuivre. Différents arguments peuvent être avancés à l'appui de la proposition d'un cadre pour conceptualiser le développement pédagogique. Tout d'abord, un tel cadre permet d'analyser et de conceptualiser les pratiques dans des contextes institutionnels variés et d'identifier ainsi celles qui sont les plus efficaces pour soutenir le développement pédagogique. Ensuite, ce cadre permet d'identifier des pistes de formation initiale et continue des personnes dont la mission est justement de soutenir, au sein des universités, le développement pédagogique des enseignants universitaires, à savoir les conseillers pédagogiques (3). Il permet également d'identifier des pistes de recherches intéressantes. Enfin il permet d'analyser l'impact de programmes de formation pédagogique, en fournissant ainsi le cadre de référence à partir duquel évaluer ces programmes.

\section{Définitions}

Au cœur même de ce cadre conceptuel, la référence au développement pédagogique des enseignants ne suscite pas l'unanimité quand il s'agit de le définir, d'autant que les significations locales et linguistiques ajoutent encore à la complexité de choisir un terme unique. Ainsi le terme même de " conseiller pédagogique " pour désigner la personne en charge, au sein d'une institution universitaire, de cette mission de formation, de conseil et d'accompagnement des enseignants, peut se révéler polysémique. L'analyse de la signification de ce terme dans différents espaces linguistiques (Di Napoli, Fry, Frenay et al., 2010) démontre, à l'instar de l'analyse systématique des publications effectuée aux États-Unis (MacDonald, 2002 ; Professional and organizational network in higher education, 2002a, 2002b; Sorcinelli, Austin, Eddy et al., 2006 ; Webb, 1996 ; Wright, 1995), qu'il n'y a pas consensus sur un terme unique et sa définition, car les différents termes employés sont éminemment contextualisés. La richesse des vocables utilisés dans la littérature anglo-saxonne pour qualifier ce champ de recherche et de pratiques montre d'ailleurs bien les dimensions spécifiques de l'expérience de la carrière d'enseignant-chercheur qui sont visées: instructional development, curriculum development, professional development, organizational development et academic development.

L'approche principalement psychopédagogique (instructional development) s'intéresse à la planification de dispositifs pédagogiques d'un enseignement, pour soutenir l'apprentissage des étudiants. Les améliorations possibles de cette approche se concentrent alors sur la définition d'objectifs et d'acquis d'apprentissage, le choix de méthodes et stratégies pédagogiques, le développement d'outils d'évaluation des acquis des étudiants et, enfin, la cohérence de ces dimensions pour améliorer l'apprentissage de chaque étudiant. L'accent est clairement mis sur la dimension pratique et sur les aspects techniques de développement des capacités de planification et d'enseignement (Mathis, 1979 ; Wilcox, 1997). C'est d'ailleurs l'approche privilégiée dans beaucoup de formations proposées aux enseignants qui arrivent au sein des universités.

L'approche par programmes (curriculum development) vise à soutenir le développement de programmes de cours (Cook, 2000), dans le mouvement d'implémentation de nouveaux curricula, faisant suite à des réformes nationales ou locales, pour répondre aux nouvelles demandes et besoins en enseignement 
supérieur. II s'agit d'identifier les nouveaux domaines scientifiques, définir les buts de formation, sélectionner et planifier des cours ou modules d'apprentissage qui soient cohérents avec le programme et qui permettent les apprentissages et les acquisitions visés par le programme, faisant en sorte de tirer le meilleur parti des ressources disponibles et supervisant l'implémentation et les résultats de ces programmes (Diamond, 1998 ; Stark \& Lattuca, 1997). Le contexte particulier du processus de Bologne, en Europe, a particulièrement mis en avant cette dimension des programmes et la demande d'un soutien à cet égard de la part des conseillers pédagogiques et des services de pédagogie universitaire (Clement, McAlpine \& Waeytens, 2004).

L'approche organisationnelle (organizational development) vise quant à elle à maximiser les ressources d'une institution pour atteindre ses objectifs et réaliser ses missions, en aidant les décideurs dans leur planification stratégique et l'implémentation de politiques à l'égard de l'enseignement et de la formation (Diamond, 2005 ; Lieberman, 2005 ; Professional and organizational network in higher education, 2002b). Les programmes de soutien au développement organisationnel se focalisent sur les politiques, structures et procédures institutionnelles qui peuvent créer des environnements (physiques, intellectuels et administratifs de l'institution) propres à développer la mission d'enseignement des universités.

L'approche professionnelle (professional development), quand ce terme est utilisé en référence au développement de la carrière des enseignants, vise chaque académique (4) comme un membre du personnel académique d'une institution universitaire et vise donc, à ce titre, son développement professionnel (Centra, 1989). En Australie, en Nouvelle-Zélande et au Royaume-Uni, le terme "academic development » est utilisé pour décrire un développement global des carrières académiques et implique l'intégration du développement personnel (professionnel et organisationnel) autour de l'ensemble des missions du travail académique (Brew \& Boud, 1996 ; Candy, 1996). Aux États-Unis, c'est le terme « faculty development " qui renvoie à cette approche globale (Professional and organizational network in higher education, 2002a). II est intéressant d'ailleurs de noter qu'audelà de l'enseignement et l'apprentissage, certains programmes proposés dans cette approche globale visent la planification de carrière, le support à l'obtention de bourses de recherche, la publication, les activités de supervision scientifique, le développement de capacités de communication et de gestion d'équipe ou de gestion du temps. Alors que l'approche professionnelle vise le seul développement d'une personne, cette approche de gestion globale inclut également l'approche organisationnelle.

\section{Processus de développement pédagogique}

La perspective retenue en finale pour notre cadre conceptuel se centre sur le développement des capacités d'enseignement et d'apprentissage des enseignants, vu comme un processus de développement pédagogique. En d'autres termes, cela fait référence à " tout le travail qui est fait systématiquement pour aider les académiques à faire de leur mieux pour soutenir l'apprentissage des étudiants " (Knight \& Wilcox, 1998). Ceci implique, du point de vue d'un académique, de réfléchir à son développement professionnel, ce qui suppose un processus en plusieurs paliers, passant de l'expertise pédagogique à l'excellence pédagogique, voire à la diffusion de celle-ci, dans la lignée des travaux sur le "scholarship of teaching and learning" (Rege Colet, McAlpine, Fanghanel et al., à paraître). Du point de vue d'une institution, penser le développement pédagogique en termes de processus, c'est fournir aux enseignants des conditions qui leur permettent de reconnaitre les besoins de leurs étudiants, d'acquérir des connaissances et des compétences en matière d'enseignement et d'apprentissage, de partager celles-ci avec leurs collègues, d'être reconnus et valorisés pour leur travail. En ce sens, le développement pédagogique (educational development) couvre toute la variété des approches décrites. II ne s'agit pas de développer une approche spécifique mais de proposer un large éventail d'activités qui concourent au développement de la mission de formation des universités et qui nécessitent de développer les synergies entre les activités et les offres de formation.

\section{LES CINQ DIMENSIONS DU CADRE CONCEPTUEL PROPOSÉ}

Le cadre conceptuel que nous proposons à la figure 1 identifie cinq dimensions principales qui permettent d'appréhender le développement pédagogique, au sens défini ci-dessus. Tout d'abord, le développement pédagogique est fondamentalement ancré dans les influences contextuelles qui dessinent le paysage de l'enseignement supérieur, ses politiques et ses pratiques. Les missions premières et la 
Figure 1. - Sens et portée du développement pédagogique : un cadre conceptuel

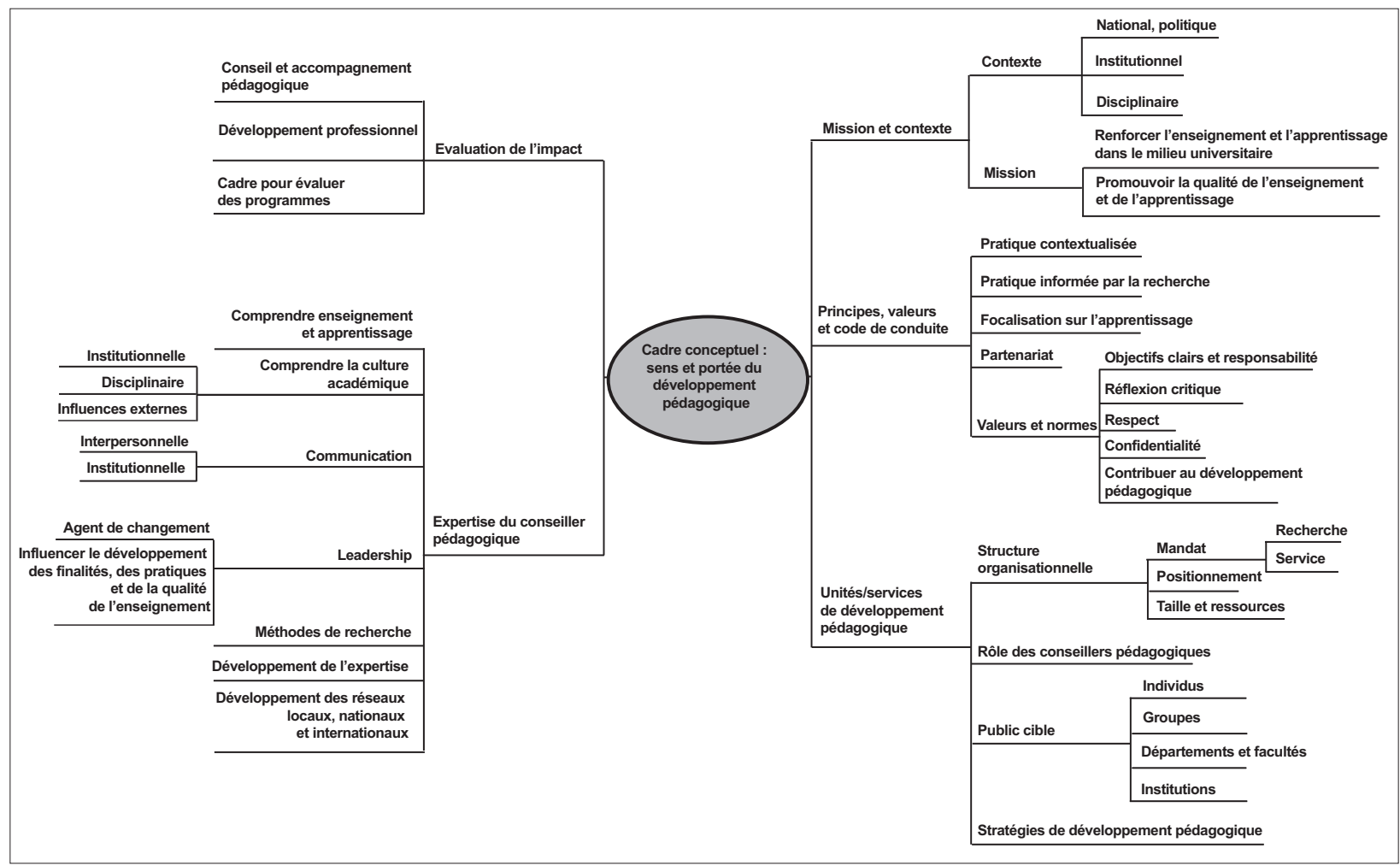

Source : Taylor \& Rege Colet, 2010, p. 158.

portée du développement pédagogique sont donc façonnées par ces contextes. Deuxièmement, on peut dégager un ensemble de principes, valeurs et normes éthiques qui définissent le conseil et l'accompagnement pédagogique pour remplir ces missions de manière adéquate. Troisièmement, la pratique de l'accompagnement et du soutien pédagogique s'inscrit dans des structures organisationnelles spécifiques qui définissent des stratégies de soutien et d'accompagnement. Quatrièmement, les pratiques de conseil et d'accompagnement du développement pédagogique s'appuient sur l'expertise des conseillers pédagogiques locaux. Enfin, cinquième et dernière dimension, la conceptualisation du conseil et de l'accompagnement du développement pédagogique et l'expertise nécessaire pour l'atteindre conduisent à rechercher des approches pertinentes, valides et fiables pour évaluer l'impact du soutien au développement pédagogique.

En choisissant une telle approche située visant à comprendre, expérimenter, développer et évaluer le développement pédagogique et ses dispositifs de soutien, il est possible de conceptualiser les cinq dimensions essentielles de ce développement pédagogique, qui transcendent les cultures locales, les institutions et les disciplines. Elles sont reprises systématiquement ci-après et explicitent le cadre, tel qu'il est schématisé par la figure 1.

\section{Mission et contexte}

Les pratiques de développement pédagogique au sein de chaque institution sont nécessairement contextualisées. Elles sont façonnées par trois types de « contextes » qui sont en interaction. D'abord, ces pratiques s'insèrent au sein d'un pays, dans un contexte national et politique qui, par la détermination des directives, lois et autres cadres nationaux plus ou moins contraignants, vont leur permettre de s'actualiser. Ensuite, à l'échelon local, au sein même de l'institution universitaire, suivant son degré d'autonomie, son histoire et ses valeurs, des documents 
politiques, des procédures locales vont orienter les priorités, les ressources et les moyens qui permettent la mise en œuvre de telle ou telle pratique. Enfin ces pratiques sont déclinées dans des environnements disciplinaires spécifiques au sein des facultés et départements qui possèdent un ensemble de buts partagés, de valeurs, de processus de recherche, d'analyse et de langage spécifique (Becher \& Trowler, 2001; Brew, 1999; Huber, 2004 ; Rice, 1996 ; Shulman, 2002 ; Swales, 1990). Le choix des pratiques de soutien au développement pédagogique des enseignants nécessite donc une appréciation fine de ces " contextes" pour les décliner localement et communiquer de manière adéquate (Dall'Alba \& Sandberg, 2006 ; Steinert, 2000 ; Taylor, 2005a). Une double mission est communément reconnue au soutien au développement pédagogique des enseignants : il s'agit à la fois de renforcer et de promouvoir la qualité de l'enseignement et de l'apprentissage des étudiants.

\section{Principes, valeurs et code de conduite}

Que ce soit les associations professionnelles de conseillers pédagogiques qui définissent les principes sous-tendant leur travail (Professional and organizational network in higher education, 2002a ; Staff and educational development network, 2005) ou les conseillers pédagogiques eux-mêmes (Di Napoli, Fry, Frenay et al., 2010), tous expriment un engagement à respecter la diversité des contextes locaux par une connaissance approfondie de ceux-ci et de leurs besoins spécifiques, une pratique informée par la recherche, par la valorisation de l'apprentissage plutôt que de l'enseignement et une collaboration entre conseillers pédagogiques et acteurs locaux (décideurs, enseignants, etc., voir notamment Candy, 1996 ; Hutchings \& Shulman, 1999 ; Scott \& Weeks, 1996 ; Steinert, 2000 ; Taylor, 2005b ; Wildman, Hable, Preston et al., 2000).

Or adopter une approche collégiale et coopérative à travers différents contextes locaux et institutionnels, tout en soutenant une approche informée par la recherche, ne va pas de soi (Knight \& Wilcox, 1998). C'est d'autant plus le cas lorsque des tensions surgissent entre les objectifs partagés par les conseillers pédagogiques ou les académiques et les objectifs institutionnels de l'université. Ou encore, lorsque les suggestions faites par des conseillers pédagogiques dans le but d'améliorer la pratique enseignante, voire lorsque les politiques proposées par les institutions universitaires pour soutenir la mission d'enseignement des académiques, vient mettre à mal les autres responsabilités de la carrière académique des enseignants-chercheurs, en réduisant par exemple le temps disponible pour faire de la recherche ou développer les services à la société. Dès lors, un code de conduite clair qui spécifie les normes et les valeurs de la profession est indispensable (comme celui proposé par le Professional and organizational network in higher education, 2002a : Ethical guidelines for educational developers). Ces normes et valeurs sont la nécessaire articulation des rôles de chacun, la remise en question de ses connaissances et compétences, le respect de l'autre (acceptation de valeurs et d'approches différentes en matière d'enseignement), la confidentialité et le partage de ses compétences.

\section{Unités et services de développement pédagogique}

Les services de développement pédagogique ou de pédagogie universitaire se différencient par les mandats institutionnels qui leur sont attribués, leur structure organisationnelle, les publics qu'ils desservent, ainsi que les rôles et stratégies de développement pédagogique qu'ils mettent en œuvre pour mener à bien leurs mandats. Suivant le contexte local, la structure organisationnelle et la manière dont les conseillers pédagogiques vont pouvoir développer leurs stratégies pédagogiques peuvent varier. On observe cependant souvent deux types de services: les services de politique éducative et les services de développement pédagogique. Les premiers sont directement concernés par la mise en œuvre de la politique d'enseignement supérieur, qui découle souvent d'orientations nationales, régionales ou institutionnelles. Ainsi, en Europe, ces services ont été largement impliqués dans l'implémentation du processus de Bologne et dans l'évaluation de la qualité de l'enseignement. Les services de développement pédagogique sont quant à eux focalisés sur le développement de la capacité d'enseignement et d'apprentissage des enseignants-chercheurs et sur les programmes de l'institution. On constate une large variété d'appellations: centres ou services de pédagogie universitaire, de soutien à l'enseignement, de développement pédagogique, etc. Dans tous les cas, on retrouve clairement l'accent mis sur l'enseignement et l'apprentissage.

Le mandat explicite de ces services de développement pédagogique inclut la planification et la coordination d'activités de formation, la dissémination de 
ressources pédagogiques, l'organisation des procédures d'évaluation de la qualité de l'enseignement et le conseil individuel aux enseignants-chercheurs ou aux commissions et responsables de programmes sur des questions associées à l'apprentissage et l'enseignement. Certains centres assument en outre le développement des technologies éducatives dans l'enseignement. Plusieurs ont également développé des fonds de soutien ou de reconnaissance des initiatives pédagogiques, qui sont sélectionnées sur la base d'un appel à projets. L'analyse des cinq études de cas détaillées dans une autre publication (Saroyan \& Frenay, 2010) permet de résumer les domaines d'activités qui font partie de la plupart des services de développement pédagogique: former, évaluer, conseiller, soutenir l'eLearning et les innovations pédagogiques et faciliter le développement de l'expertise pédagogique (Frenay \& Saroyan, 2010). L'importance que prend chacun de ces domaines d'activités varie d'un centre à l'autre et dépend des mandats institutionnels explicites et des ressources disponibles.

\section{Structure organisationnelle}

Les services ou centres de développement pédagogique sont habituellement des unités centralisées, directement attachées au recteur ou au président en charge de l'enseignement et de la formation. Une telle place dans la structure organisationnelle positionne ces services différemment des départements académiques et les situe davantage comme des services administratifs ou de support, au même titre que d'autres services universitaires, comme les services multimédias et informatiques, les services financiers ou les services en charge du personnel. Cependant, comme la plupart de ces services visent à disséminer et à construire des connaissances basées sur la recherche et l'expertise pour améliorer l'enseignement et l'apprentissage, le travail de développement pédagogique nécessite une approche académique, même là où le mandat ne prévoit pas explicitement que la recherche fasse partie des missions du service. Quelle que soit la stratégie choisie, l'idée est de construire des ponts entre le niveau central et les niveaux locaux de développement pédagogique. Ces collaborations peuvent déboucher sur des projets de recherche conjoints ou sur l'organisation d'activités ou de conseil communs et impliquer des enseignantschercheurs dans le travail de ces services.

Peu d'institutions universitaires ont les ressources pour implanter de tels services de développement pédagogique au sein de chaque faculté, même si les enseignants-chercheurs et les conseillers pédagogiques pointent à juste titre la nécessité d'un développement pédagogique ancré dans l'approche disciplinaire spécifique. On peut cependant noter que les facultés de médecine font souvent exception à cette observation, car elles ont souvent développé leur propre unité de pédagogie médicale. Des évolutions récentes constatées dans certaines institutions montrent à quel point cette question est fondamentale (Clement \& Frenay, 2010).

\section{Public cible}

L'analyse comparative des études de cas (Frenay \& Saroyan, 2010) a permis de montrer qu'en général, les services de développement pédagogique sont accessibles à tout membre de la communauté académique mais que, souvent, les enseignants-chercheurs nouvellement engagés constituent le public cible le plus important. En effet ces collègues qui démarrent dans la carrière avec peu de formation pédagogique, tout en étant confrontés à des responsabilités d'enseignement, sont souvent motivés pour participer à des programmes de formation et pour ainsi obtenir du soutien face aux défis auxquels ils sont confrontés. Ceci explique sans doute le fait que de nombreux programmes de formation sont explicitement organisés pour les enseignants-chercheurs en début de carrière, pour les introduire sur une trajectoire de développement professionnel qui combine recherche et enseignement, plutôt que de maintenir une distinction nette entre leurs deux activités principales (Austin, 2002 ; Gaff, 2002). D'autres publics sont visés : les chargés de cours, qui n'ont pas de charge permanente à l'université (Gappa \& Leslie, 1993 ; Lyons, 2004), et les doctorants, pour qui des activités spécifiques de développement pédagogiques sont proposées. Ces activités peuvent prendre la forme d'ateliers, de séminaires, de moments d'accompagnement, de cours formels intégrés au parcours de formation doctorale (Marincovich, Prostko \& Stout, 1998 ; Schönwetter \& Taylor, 2003), ou encore de programmes débouchant sur un diplôme spécifique (comme par exemple un master en pédagogie de l'enseignement supérieur).

Dans certaines universités, les enseignantschercheurs plus expérimentés constituent un autre groupe cible. Ces personnes restent souvent intéressées par l'enseignement mais ont peu l'occasion de partager leurs points de vue avec d'autres collègues, ou d'essayer de nouvelles initiatives dans leurs classes (LaCelle-Peterson \& Finkelstein, 1993 ; Romano, Hoesing, O'Donovan et al., 2004). Karpiak (1997) soulignait l'isolement de la mi-carrière, l'importance de rester partie prenante d'une communauté académique 
et celle de garder le sentiment de réaliser un travail qui compte. Blaisdell et Cox (2004) ont ainsi mis en place une expérience pour engager à nouveau les collègues expérimentés dans des communautés d'enseignants-chercheurs où ils peuvent profiter de l'expérience collective et s'impliquer dans un processus de développement pédagogique. Ces collègues sont également souvent bien placés pour participer de manière constructive à des consultations auprès de leurs pairs, pour du "mentorat ", des groupes de discussion ou encore des réformes de programmes.

\section{Rôles et stratégies d'accompagnement}

II existe toute une série de stratégies d'accompagnement pour remplir les missions assignées aux services de développement pédagogique. Cela peut aller d'ateliers uniques à des programmes complets ou des masters en pédagogie universitaire. Des programmes de mentorat sont également souvent disponibles pour soutenir les enseignants novices et développer leurs pratiques pédagogiques via des contacts réguliers avec des enseignants-chercheurs plus expérimentés. Les services de développement pédagogique offrent également des consultations individuelles ou en équipe. Les consultations individuelles sont souvent centrées sur la planification d'un cours, les méthodes pédagogiques, le lancement et la mise en œuvre d'innovations, l'usage du eLearning, ou encore l'évaluation des étudiants. Elles peuvent également mener à des observations en classe portant sur les compétences d'enseignement.

Au niveau d'un programme ou d'un département, les rencontres menées avec un conseiller pédagogique peuvent se centrer sur les réformes des programmes, leur évaluation, la manière d'allouer les ressources pédagogiques, d'évaluer la charge de travail des étudiants, d'augmenter la collaboration ou encore d'ajuster les méthodes d'évaluation des étudiants. Les conseillers pédagogiques peuvent ainsi fournir conseil ou accompagnement suite à une évaluation de cours ou de programmes, voire participer à la préparation des évaluations externes ou des accréditations.

Au niveau plus institutionnel, les conseillers pédagogiques sont sollicités pour construire les politiques pédagogiques et les procédures qui permettent de valoriser et reconnaître l'engagement des enseignantschercheurs dans leur enseignement. Ils contribuent ainsi à influencer la manière dont la performance d'un enseignant est évaluée ou reconnue à travers les procédures de promotion ou de confirmation (5). S'il est indispensable de contribuer à construire l'infrastruc- ture administrative et physique qui soutienne l'enseignement pour développer la capacité individuelle ou institutionnelle portant sur l'apprentissage et la formation, de même il est indispensable de penser une politique de communication qui fournisse de manière efficace les informations à propos des ressources, des politiques et de leur implémentation, etc. Ainsi, dans les cinq études de cas analysées (Saroyan \& Frenay, 2010), les conseillers pédagogiques jouent un rôle de relais afin que ces informations atteignent effectivement les publics visés. À travers toutes ces fonctions, le travail des conseillers pédagogiques est plus souvent situé dans la sphère académique et nécessite notamment une posture de chercheur. Cependant, quelles que soient les tâches qu'ils réalisent, les conseillers pédagogiques peuvent endosser des rôles différents, qui sont le reflet de la manière dont ils estiment devoir assumer leur fonction de conseil. Ils peuvent ainsi davantage se positionner en expert dans la situation ou encore en partenaire, voire en formateur. Des recherches récentes ont ainsi mis en évidence une grande diversité dans les rôles que pouvaient tenir les conseillers pédagogiques (Di Napoli, Fry, Frenay et al., 2010 ; Land, 2004). Quelle que soit la position académique ou administrative qu'ils occupent, les conseillers pédagogiques doivent être engagés dans un travail académique pour la crédibilité et la validité de leurs interventions (Taylor, 2005a). Idéalement, leur travail devrait être caractérisé par un processus de recherche partagé avec les collègues, dont le but ultime serait bien de créer des situations d'apprentissage plus efficaces pour les étudiants (Rege Colet, McAlpine, Fanghanel et al., à paraître).

\section{Expertise du conseiller pédagogique}

Malgré la complexité de la pratique du développement pédagogique et du métier de conseiller pédagogique, il n'existe que peu de programmes de formation pour devenir un conseiller pédagogique. Beaucoup de ces derniers s'engagent dans une telle fonction sans formation spécifique. Ainsi une enquête menée en 2006 (Germain-Rutherford \& Grandtner, 2006) auprès de 50 conseillers pédagogiques qui travaillent au Québec ou dans des universités francophones européennes a révélé que la majorité d'entre eux détiennent un diplôme de $2^{\mathrm{e}}$ cycle (master) en psychologie ou en sciences de l'éducation. Cependant un tiers de ces conseillers arrivent à ce métier à partir d'un champ disciplinaire tout à fait différent. L'expertise dans ce métier s'acquiert essentiellement à partir de toutes les expériences vécues. Or une 
enquête menée aux États-Unis a montré que, sur environ 500 répondants, plus de la moitié d'entre eux avaient moins de cinq années d'expérience comme conseiller pédagogique (Sorcinelli, Austin, Eddy et al., 2006). Un tel type de profil, associé au fait que la pratique du développement pédagogique possède un large spectre, suggère qu'il serait utile que des programmes de formation initiale et continue puissent être organisés à destination des conseillers pédagogiques. Les domaines dans lesquels ils devraient développer une certaine expertise et des compétences importantes se situent autour de trois axes: les processus d'enseignement et d'apprentissage, la culture académique et les cultures disciplinaires et le domaine du leadership et du changement dans les organisations.

Ainsi, de nombreux auteurs s'accordent pour souligner que le travail des conseillers pédagogiques repose sur une connaissance approfondie des processus d'apprentissage et d'enseignement, en maîtrisant, voire en menant des recherches sur les différentes dimensions des pratiques enseignantes, sur les approches pour préparer les enseignants à soutenir de manière efficace l'apprentissage des étudiants, sur l'apprentissage et la motivation des étudiants, et sur les variables qui soutiennent le processus d'apprentissage (Sorcinelli, Austin, Eddy et al., 2006). Les travaux menés à l'Université McGill notamment ont ainsi bien mis en évidence que la connaissance des modèles de développement professionnel et de développement de l'expertise en situation professionnelle est nécessaire pour adopter des approches professionnelles, théoriquement et empiriquement documentées (Saroyan \& Amundsen, 2004).

Leur positionnement institutionnel, plus souvent central que local, et les contacts réguliers qu'ils entretiennent avec les facultés permettent aux conseillers pédagogiques de développer une bonne appréhension de la culture académique. Ceci est important puisque l'on sait que les différences institutionnelles et disciplinaires sont souvent prises en compte dans la manière de développer les projets pédagogiques et les activités d'enseignement (Huber, 2004 ; Becher \& Trowler, 2001; Hativa \& Marincovich, 1995; Middendorf \& Pace, 2004 ; Neumann, Parry \& Becher, 2002 ; Shulman, 1987). À ce titre, on peut considérer que les services de pédagogie universitaire ou de développement pédagogique sont des observatoires particulièrement intéressants de la vie académique et qu'ils possèdent des informations uniques sur les traditions institutionnelles et disciplinaires. Comment les cultures disciplinaires affectent-elles l'enseignement et les croyances personnelles des enseignants ? Quel est le poids des traditions départementales et des habitudes en matière d'enseignement? Pour aider non seulement les individus, mais également les équipes et les responsables en charge de questions pédagogiques, un savoir ciblé sur le leadership et la manière dont il fonctionne dans des situations particulières est très pertinent (Taylor \& Schönwetter, 2002). Les conseillers pédagogiques peuvent ainsi souvent identifier les principaux acteurs de changement organisationnel et leur propre rôle de leadership pour promouvoir et soutenir le développement de l'enseignement dans les communautés académiques. Ils peuvent se servir de cette base de connaissances pour soutenir les stratégies institutionnelles et encourager les innovateurs et les responsables pédagogiques. Comme l'a observé Taylor (2005b, p. 43-44), les conseillers réalisent un leadership efficace quand ils possèdent une large gamme de compétences telles que l'écoute, l'appréhension des problèmes et des contextes locaux, la planification ou la mise en œuvre d'activités de formation et l'établissement de partenariats permettant l'autonomie progressive des acteurs locaux (Kouzes \& Posner, 1995; Ramsden, 1998).

À travers les études de cas (Saroyan \& Frenay, 2010), nous avons observé que les contacts réguliers qu'entretiennent les conseillers pédagogiques avec les autorités académiques et administratives de leur institution leur permettent d'être partie prenante de la politique institutionnelle en matière d'enseignement et de formation. De manière générale, les conseillers pédagogiques doivent ainsi également avoir des références solides en matière de changement : comment soutenir et faciliter le changement à l'intérieur d'une organisation, comment donner des conseils judicieux sans pour autant aliéner l'équipe d'enseignants, comment amener chacun à un but commun, comment guider un travail collectif, soutenir l'apprentissage de l'équipe enseignante et gérer des projets à grande échelle? Analyser l'expertise des conseillers pédagogiques permet d'identifier les compétences et capacités qui constituent leur pratique professionnelle. Une telle analyse rend possible la construction d'un cadre pour initier une formation initiale et continue en développement pédagogique et ainsi proposer des objectifs pour de tels programmes (Staff and educational development network, 2005). Alors que de plus en plus d'universités proposent des cours de niveau master en pédagogie universitaire aux doctorants ou aux jeunes enseignants-chercheurs, il n'existe que très peu d'occasions d'avoir une formation académique portant sur le conseil pédagogique. 
C'est donc vers la formation continue que se tournent les conseillers pédagogiques, notamment par les échanges de pratiques entre pairs, nouveaux ou plus expérimentés. Comme dans d'autres professions, le développement pédagogique des conseillers pédagogiques se fait au travers de la participation active à des congrès, des ateliers et des programmes de formation professionnelle. C'est d'autant plus important dans un tel contexte que des réseaux professionnels existent à différents niveaux (régionaux, nationaux, internationaux), par le biais d'associations professionnelles ou de recherche et de leurs outils de communication.

\section{Évaluation de l'impact de l'accompagnement du développement pédagogique}

L'hypothèse sous-jacente à la mise en place de programmes de développement pédagogique est bien que ce dernier est susceptible d'avoir un impact positif sur le développement de la capacité d'apprendre et d'enseigner. Dès lors, le défi le plus important relevé dans nos études de cas et auxquels les conseillers pédagogiques sont confrontés est de pouvoir développer des recherches dont les résultats démontrent l'impact de ces programmes et activités. II s'agit de tester s'il existe bien une relation entre les activités suivies et les changements survenus, tant au niveau des conceptions que des pratiques enseignantes. Quels résultats démontrent que le développement pédagogique est efficace et qu'il atteint les buts que lui ont assignés à la fois l'institution et les conseillers pédagogiques (Knight \& Wilcox, 1998) ? Quels résultats soutiennent que ce processus est efficient, vaut son investissement et se justifie dans un contexte de rareté de ressources?

Or, aujourd'hui, les recherches qui mesurent les effets des programmes de développement pédagogiques sont trop rares (Chism \& Szabo, 1998 ; Steinert, 2000 ; Weimer \& Lenze, 1991), voire ne développent pas une méthodologie suffisamment solide, ou encore leurs résultats ne sont pas diffusés via les canaux traditionnels de la recherche, les revues scientifiques (Glassick, Huber \& Maeroff, 1997). De plus, les services de pédagogie universitaire ou de développement pédagogique seront soumis dans l'avenir à des procédures internes d'assurance qualité, voire à des évaluations externes, ce qui pourrait donner, s'il en était besoin, un argument supplémentaire à cette nécessité de mesure de cet impact. Le fait même de créer des réseaux à un niveau international pourrait soutenir la mise en œuvre de programmes de recherches appropriés pour évaluer l'efficacité du développement pédagogique, mais aussi, ce faisant, pour développer les modèles théoriques et les contributions empiriques qui feront du développement pédagogique un champ de connaissances et de pratiques au sein de la pédagogie universitaire.

\section{CONCLUSION : DÉFIS POUR LA PROFESSION ET LA RECHERCHE EN DÉVELOPPEMENT PÉDAGOGIQUE}

Partant de ce projet collaboratif, de ses résultats, du cadre que nous venons de présenter, nous voudrions souligner enfin les défis majeurs que nous retenons et qui se déclinent ensuite au niveau de la pratique et de la recherche sur le conseil et l'accompagnement du développement pédagogique. Comment établir et améliorer le champ de recherches et de pratiques dans le domaine du développement pédagogique, de telle manière que ce champ devienne essentiel à la résolution des besoins urgents venant des transformations continuelles de l'enseignement supérieur? Comment faire en sorte que la pratique du conseil pédagogique devienne plus crédible et mieux informée par la recherche? Pour cette pratique, nous identifions ainsi quatre défis majeurs :

- la reconnaissance large par la communauté académique de la pertinence et de la valeur de l'accompagnement pédagogique ;

- le fait de pouvoir gérer les tensions qui surviennent de divergences dans la conception de la qualité de la formation poursuivie par différents groupes d'acteurs (académiques, étudiants, gestionnaires, parents, employeurs, etc.) ou à un manque de cohérence entre les objectifs de la formation, les politiques institutionnelles qui les définissent et les ressources disponibles ;

- les attentes changeantes au regard de la mission d'enseignement des universités et des rôles et responsabilités de la communauté académique, suite aux modifications importantes qui se sont produites dans les systèmes d'enseignement supérieur ces dernières années (obligation de rendre des comptes, diversité des étudiants, nouvelles technologies, limitation des ressources pour l'enseignement supérieur). Un environnement mouvant combiné avec des budgets plus serrés, mais une exigence plus forte en termes de qualité de la formation conduit à augmenter le niveau de tensions dans le système ; 
- la construction de communautés académiques pour soutenir le travail pédagogique. Ces communautés devraient être caractérisées par des objectifs et valeurs partagés, des processus de recherche et de mise en critique, de réflexion et d'analyse (feedback entre pairs, débats) et une capacité à construire collectivement les connaissances du domaine (Huber, 2004 ; Swales, 1990).

Pour la recherche sur le développement pédagogique, on peut identifier également les principaux défis sur le long terme. Le défi le plus important est certainement de valider les interventions les plus efficaces qui permettront de proposer des formations pédagogiques portant sur les méthodes éducatives et l'évaluation et dont on peut mesurer l'impact sur l'apprentissage des enseignants-chercheurs, mais aussi sur l'apprentissage des étudiants. Plusieurs pistes de recherche peuvent être suggérées pour travailler en ce sens : quelles sont les mesures d'impact à utiliser ? Comment mesurer l'impact à long terme de l'accompagnement pédagogique? La mise en œuvre d'études d'impact ne risque-t-elle pas d'influencer le climat et la nature même du travail mené avec des enseignants-chercheurs ? Comment mesurer les effets médiateurs et modérateurs des différentes variables et processus impliqués ? Sachant que le processus de développement professionnel d'un enseignant-chercheur prend du temps, comment suivre de manière longitudinale tous les enseignantschercheurs? Comment associer les interventions des conseillers pédagogiques aux effets observés? Compte tenu du fait qu'il ne s'agit pas le plus souvent d'interventions uniques, quelles données recueillir? Quel type de recherche permettrait d'atteindre cet objectif? Quels sont les facteurs qui soutiennent ou minent la construction de communautés de pratiques chez les enseignants-chercheurs ?

En conclusion, on peut retenir de l'expérience de notre partenariat et sur la base du cadre conceptuel qui a été présenté tout au long de cet article qu'il est indispensable, d'une part que la pratique de conseil et d'accompagnement pédagogique soit ancrée dans des cadres de référence théoriques solides, et que, d'autre part, la recherche menée ait un haut potentiel d'applications pratiques et soit largement diffusée. En outre, comme c'est un champ de pratiques, l'accompagnement du développement pédagogique doit à la fois être pertinent et efficace, durable et exercé sur le moyen, voire le long terme. Ceci nécessite que des recherches soient également menées pour mesurer son impact et son efficacité à court, moyen et long termes.

Mariane Frenay mariane.frenay@uclouvain.be

GIRSEF, Chaire UNESCO de pédagogie universitaire, Université catholique de Louvain, Belgique

Alenoush Saroyan Department of educational and counselling psychology, McGill University, Canada

K. Lynn Taylor Center for learning and teaching, Dalhousie University, Canada

Denis Bédard

Centre d'études et de recherche en enseignement supérieur, Université de Sherbrooke, Canada

Mieke Clement Centre for educational development, Katholieke Universiteit Leuven, Louvain, Belgique

Nicole Rege Colet Dipartimento formazione e apprendimento, SUPSI, Locarno, Suisse Jean-Jacques Paul IREDU, université de Bourgogne, France

Anette Kolmos UNESCO Chair in Problem based learning in engineering education, Université d'Aalborg, Danemark

\section{NOTES}

(1) Les pratiques d'accompagnement et de conseil pédagogique de cinq pays (Canada, Suisse, Belgique, Danemark et France) ont fait l'objet d'une analyse approfondie dans l'ouvrage de Saroyan et Frenay (2010). L'objectif était ainsi de dresser, dans cinq chapitres distincts, le panorama critique des pratiques de développement pédagogique proposées dans les institutions universitaires pour chacun de ces pays (pris chacun comme une étude de cas) et de les resituer dans leurs contextes locaux, régionaux et nationaux. En outre, dans un chapitre comparatif (Frenay \& Saroyan, 2010), nous établissons une analyse comparative du développement de ces pratiques dans les cinq pays, en analysant notamment les modes de coordination à l'œuvre (en nous basant sur la typologie de Hollingsworth \& Boyer, 1997, adaptée pour l'éducation par Maroy \& Dupriez, 2000) et la façon dont ces modes de coordination interagissent pour faconner les pratiques proposées. Enfin nous proposons une lecture systématique et comparative, pour ces pays, des unités ou services de pédagogie universitaire en distinguant la structure organisationnelle de ces services au sein des universités, leur mandat institutionnel et leur offres d'activités, les publics cibles des actions, ainsi que les rôles et stratégies de développement pédagogique préconisés.

(2) Un des résultats sans doute les plus fondamentaux du projet évoqué, pour tous les partenaires du projet et bien sûr pour les 
auteurs de l'article, est le fait d'avoir consolidé notre compréhension de notre champ de recherche et de pratiques. Ceci s'est produit à travers ce que Bransford et ses collègues appellent "searching for a conceptual collision" (Bransford, Stevens, Schwartz et al., 2006, p. 229). Nos différentes perspectives à propos du conseil, de la formation, du soutien et de l'accompagnement pédagogique nous ont aidées, au départ, à mettre à plat nos présupposés tacites et à les comparer. C'est dans ce processus que se sont construites nos compréhensions nouvelles du sens et de la portée du concept de "faculty development » qui, au fil du temps, a davantage été considéré comme "educational development " et qui se traduit dans le cadre conceptuel développé et explicité ici. Nous avons aussi identifié progressivement les tensions qui sous-tendent la pratique du développement pédagogique, les défis posés à chaque pays, mais aussi au champ de recherches et de pratiques de la pédagogie universitaire.

(3) Les conseillers pédagogiques dont la mission principale, au sein des universités, est d'organiser les services offerts aux enseignants pour se développer pédagogiquement portent, selon les universités, des appellations différentes mais qui toutes traduisent l'idée que ces personnes accompagnent, soutiennent les enseignants, les équipes d'enseignants, les responsables de programmes ou les responsables institutionnels pour leur permettre d'améliorer la qualité de leur offre de formation, à travers différentes actions de formation ou de conseil (voir Di Napoli, Fry, Frenay et al., 2010).

(4) Dans le monde universitaire belge, le terme « enseignantchercheur " n'est pas usité comme tel. Quand on fait référence à cette catégorie de personnel, on parle du personnel académique, c'est-à-dire le personnel qui assume les fonctions d'enseignantchercheur au sens français, à savoir les trois missions classiquement assignées à l'université, les missions d'enseignement, de recherche et de service à la société. Par extension, un académi- que est une personne qui occupe un poste académique ou qui poursuit une carrière académique, ou encore qui est membre du corps académique de l'université, qu'il ait le titre de professeur ordinaire, professeur ou chargé de cours. En Suisse, on parle du corps professoral qui occupe les postes académiques, soit comme professeur ordinaire, associé ou chargé de cours. Aux États-Unis et au Canada, c'est le terme " faculty » qui est utilisé dans le même sens: l'ensemble des personnes qui occupent les postes académiques au sein des facultés, à avoir qui exercent des missions d'enseignement, de recherche et de service (avec le titre de full professor, associate professor ou assistant professor). C'est pourquoi on retrouve souvent la notion de "faculty development" dans la littérature anglo-saxonne, qui renvoie à l'idée qu'on vise le développement de ces académiques, engagés à la fois pour des missions d'enseignement, de recherche et de services à la société. En Australie, en NouvelleZélande et au Royaume-Uni, on parle d'" academic development » pour faire référence à cette même idée.

(5) Dans la carrière académique classique, au Canada, en Belgique, au Danemark et aux États-Unis notamment, les enseignantschercheurs démarrent dans une fonction temporaire, comme " assistant professor » ou " chargé de cours temporaire » et ne sont "confirmés» dans leur poste académique qu'après 3 à 5 ans, après avoir déposé un dossier de confirmation qui est évalué par une commission spécifique. De même, pour ensuite changer de rang dans la carrière et atteindre le rang de professeur, professeur ordinaire ou professeur titulaire (au Québec), ou encore «associate professor", "full professor » au Canada et aux États-Unis, les enseignants-chercheurs doivent déposer un dossier de demande de promotion qui fait état de leur avancement en matière de recherche, d'enseignement et de services à la société. Une commission spécifique constituée de pairs désignés par le rectorat évalue les dossiers et propose ou non la candidature de l'enseignant à la promotion demandée.

\section{BIBLIOGRAPHIE}

AUSTIN A. (2002). "Creating a bridge to the future: Preparing new faculty to face changing expectations in a shifting context ». Review of Higher Education, vol. 26, $\mathrm{n}^{\circ} 2$, p. 119-144.

BECHER T. \& TROWLER P. (2001). Academic tribes and territories: Intellectual inquiry and the cultures of discipline. Buckingham: Society for research into higher education et Open University Press.

BÉDARD D., CLEMENT M. \& TAYLOR K. L. (2010). «Validation of a conceptual framework on faculty development: Meaning and scope ". In A. Saroyan \& M. Frenay (dir.), Building teaching capacities in higher education: A comprehensive international model. Sterling: Stylus Publishing, p. 168-187.

BLAISDELL M. \& COX M. (2004). « Midcareer and senior faculty learning communities: Learning throughout faculty careers ». In M. Cox \& L. Richlin (dir.), Building faculty learning communities. $N^{\circ}$ 97: New directions for teaching and learning. San Francisco: Jossey-Bass, p. 137-148.

BRANSFORD J., STEVENS R., SCHWARTZ D. et al. (2006). "Learning theories and education: Toward a decade of synergy ». In P. Alexander \& P. Winnie (dir.), Handbook of educational psychology. Mahwah : Erlbaum, p. 209244.

BREW A. (1999). "Research and teaching: Changing relationships in a changing context ". Studies in Higher Education, vol. 24, n³, p. 291-301.
BREW A. (2002). «Research and the academic developer: A new agenda ». International Journal for Academic Development, vol. 7, $\mathrm{n}^{\circ}$ 2, p. 112-122.

BREW A. \& BOUD D. (1996). "Preparing for new academic roles: An holistic approach to development ». International Journal for Academic Development, vol. 1, $\mathrm{n}^{\circ} 2$, p. 17-25.

CANDY P. (1996). "Promoting life-long learning: Academic developers and the university as a learning organization ». International Journal for Academic Development, vol. $1, n^{\circ} 1$, p. 7-18.

CENTRA J. (1989). «Faculty evaluation and faculty development in higher education ». In J. Smart (dir.), Higher education: Handbook of theory and research. New York: Agathon Press, p. 155-179.

CHISM N. \& SZABO B. (1998). "How faculty development programs evaluate their services ». Journal of Staff, Program \& Organizational Development, vol. $15, \mathrm{n}^{\circ} 2$, p. 55-62.

CLEMENT M. \& FRENAY M. (2010). « Faculty development in Belgian universities ». In A. Saroyan \& M. Frenay (dir.), Building teaching capacities in higher education: A comprehensive international model. Sterling: Stylus Publishing, p. 82-103.

CLEMENT M., McALPINE L. \& WAEYTENS K. (2004). «Fascinating Bologna: Impact on the nature and approach of academic development ". International Journal for Academic Development, vol. 9, $\mathrm{n}^{\circ}$ 2, p. 127-131. 
COOK C. (2000). " The role of a teaching centre in curricular reform ». In D. Lieberman \& C. Wehlburg (dir.), To improve the academy: Resources for faculty, instructional, and organizational development, vol. 19. Bolton: Anker, p. 217-231.

DALL'ALBA G. \& SANDBERG J. (2006). « Unveiling professional development: A critical review of stage models ". Review of Educational Research, vol. 76, n 3, p. 383412.

DIAMOND R. (1998). Designing and assessing courses and curricula: A practical guide. San Francisco: JosseyBass.

DIAMOND R. (2005). "The institutional change agency: The expanding role of academic support centers ». In S. Chadwick-Blossey \& D. Robertson (dir.), To improve the academy: Resources for faculty, instructional, and organizational development, vol. 23. Bolton : Anker, p. 24-37.

DI NAPOLI R., FRY H., FRENAY M., VERHESSCHEN P. \& VERBURGH A. (2010). "Academic development and educational developers: perspectives from different European higher education contexts ". International Journal for Academic Development, vol. 15, $\mathrm{n}^{\circ} 1$, p. 7-18.

FRENAY M. \& SAROYAN A. (2010). «Faculty development across Europe and Canada: Comparisons of five case studies ». In A. Saroyan \& M. Frenay (dir.), Building teaching capacities in universities: A comprehensive international model. Sterling : Stylus Publishing, p. 111-138.

FRENAY M., SAROYAN A., CLEMENT M. et al. (2005). FACDEV Program: Promoting faculty development to enhance the quality of learning in higher education. Bruxelles : Union européenne.

GAFF J. (2002). "The disconnect: Graduate education and faculty realities. A review of recent research ». Liberal Education, vol. 88, n³, p. 6-13.

GAPPA J. \& LESLIE D. (1993). The invisible faculty: Improving the status of part-timers in higher education. San Francisco : Jossey-Bass.

GERMAIN-RUTHERFORD A. \& GRANDTNER A. (2006). "Professional development for educational developers: The BSQ model ". Annual conference of the Educational developers caucus of the Society for teaching and learning in higher education.

GLASSICK C., HUBER M. \& MAEROFF G. (1997). Scholarship assessed: Evaluation of the professoriate. San Francisco : Jossey-Bass.

HATIVA N. \& MARINCOVICH M. (1995). New directions for teaching and learning. Vol. 64: Disciplinary differences in teaching and learning: Implications for practice. San Francisco: Jossey-Bass, p. 1-4.

HOLLINGSWORTH J. \& BOYER R. (1997). Contemporary capitalism: The embededdness of institutions. Cambridge : Cambridge University Press.

HUBER M. (2004). Balancing acts: The scholarship of teaching and learning in academic careers. Washington: American association for higher education et Carnegie foundation for the advancement of teaching.

HUTCHINGS P. \& SHULMAN L. (1999). "The scholarship of teaching: New elaborations, new developments ". Change, vol. $31, n^{\circ} 5$, p. 10-15.
KARPIAK I. (1997). «University professors at mid-life: Being part of... but feeling apart ». In D. Dezure (dir.), To improve the academy: Resources for faculty, instructional, and organizational development, vol. 16. Bolton: Anker, p. 21-40.

KNIGHT P. \& WILCOX S. (1998). «Effectiveness and ethics in educational development: Changing contexts, changing notions ». International Journal for Academic Development, vol. 3, $\mathrm{n}^{\circ} 2$, p. 97-106.

KOUZES J. \& POSNER B. (1995). The leadership challenge. San Francisco : Jossey-Bass.

LACELLE-PETERSON M. \& FINKELSTEIN M. (1993). «Institutions matter: Campus teaching environments' impact on senior faculty ". In M. Finkelstein \& M. LaCellePeterson (dir.), New directions for teaching and learning, $n^{\circ}$ 55: Developing senior faculty as teachers. San Francisco : Jossey-Bass, p. 21-32.

LAND R. (2004). Educational development: Discourse, identity and practice. Maidenhead : Open University Press.

LEVINSON-ROSE J. \& MENGES R. (1981). «Improving college teaching: A critical review of research ». Review of Educational Research, vol. 51, n³, p. 403-434.

LIEBERMAN D. (2005). "Beyond faculty development: How centers for teaching and learning can be laboratories for learning ». In A. Kezar (dir.), New directions for higher Education, $n^{\circ}$ 131: Organizational learning in higher education. San Francisco : Jossey-Bass, p. 87-98.

LYONS R. (2004). Success strategies for adjunct faculty. Boston : Pearson.

MacDONALD R. (2002). «Academic development: Research, evaluation and changing practice in higher education ". In R. MacDonald \& J. Wisdom (dir.), Academic and educational development: Research, evaluation and changing practice in higher education. Londres: Falmer Press, p. 3-13.

MARINCOVICH M., PROSTKO J. \& STOUT F. (1998). The professional development of graduate teaching assistants. Bolton : Anker.

MAROY C. \& DUPRIEZ V. (2000). «La régulation dans les systèmes scolaires. Proposition théorique et analyse du cadre structurel en Belgique francophone ». Revue française de pédagogie, $\mathrm{n}^{\circ} 130$, p. 73-87.

MATHIS B. (1979). «Faculty development». In S. Ericksen \& J. Cook (dir.), Support for teaching at major universities. Ann Arbor: Center for research on learning and teaching, University of Michigan.

MIDDENDORF J. \& PACE D. (2004). « Decoding the disciplines: A model for helping students learn disciplinary ways of thinking ». In D. Pace \& J. Middendorf (dir.), New directions for teaching and learning. Vol. 98: Decoding the disciplines: Helping students learn disciplinary ways of thinking. San Francisco : Jossey-Bass, p. 1-12.

NEUMANN R., PARRY S. \& BECHER T. (2002). « Teaching and learning in their disciplinary contexts: A conceptual analysis ". Studies in Higher Education, vol. 27, $n^{\circ} 4$, p. 405-417.

PROFESSIONAL AND ORGANIZATIONAL NETWORK IN HIGHER EDUCATION (2002a). Ethical guidelines for educational developers. Disponible sur Internet à l'adresse : <http://www.podnetwork.org/faculty_development/ethicalguidelines.htm> (consulté le 6 octobre 2010). 
PROFESSIONAL AND ORGANIZATIONAL NETWORK IN HIGHER EDUCATION (2002b). What is faculty development. Disponible sur Internet à l'adresse : <http://www. podnetwork.org/development.htm> (consulté le 6 octobre 2010).

RAMSDEN P. (1998). Learning to lead in higher education. Londres : Routledge.

REGE COLET N., McALPINE L., FANGHANEL J. \& WESTON C. (à paraître). "La recherche liée à l'enseignement au supérieur et la formalisation des pratiques enseignantes : le concept du SoTL ". Recherche et formation.

RICE R. (1996). Making a place for the new American scholar. Washington: American association for higher education.

ROMANO J., HOESING R., O'DONOVAN K. \& WEINSHEIMER J. (2004). «Faculty at mid-career: A program to enhance teaching and learning ". Innovative Higher Education, vol. $29, n^{\circ} 1$, p. $21-48$.

ROWLAND S. (1999). "The role of theory in a pedagogical model for lecturers in higher education ". Studies in Higher Education, vol. 24, n³, p. 303-314.

SAROYAN A. \& AMUNDSEN C. (2004). Rethinking teaching in higher education: From a course design workshop to a faculty development framework. Sterling: Stylus Publishing.

SAROYAN A. \& FRENAY M. (2010). Building teaching capacities in higher education: A comprehensive international model. Sterling : Stylus Publishing.

SCHÖNWETTER D. \& TAYLOR K. L. (2003). «Preparing future professors for their teaching roles: Success strategies from a Canadian program ". Journal of Teaching Assistant Development, vol. 9, n¹, p. 101-110.

SCOTT D. \& WEEKS P. (1996). " Collaborative staff development». Innovative Higher Education, vol. 21, $\mathrm{n}^{\circ} 2$, p. 101-111.

SELL G. \& CHISM N. (1991). " Finding the right match: Staffing faculty development centers ». In K. Zahorski (dir.), To improve the academy: Resources for faculty, instructional, and organizational development, vol. 10. Bolton : Anker, p. 19-29.

SHULMAN L. (1987). "Knowledge and teaching: Foundations of the new reform ". Harvard Educational Review, vol. $36, \mathrm{n}^{\circ} 1$, p. 1-22.

SHULMAN L. (2002). «Making differences: A table of learning ". Change, vol. 34, n 6 , p. 36-44.

SORCINELLI M., AUSTIN A., EDDY P. \& BEACH A. (2006). Creating the future of faculty development: Learning from the past, understanding the present. Bolton : Anker.

STAFF AND EDUCATIONAL DEVELOPMENT NETWORK (2005). Professional development framework. Disponi- ble sur Internet à l'adresse: <http://www.seda.ac.uk/ professional-development.html?p=3 1> (consulté le 4 octobre 2010).

STARK J. \& LATTUCA J. (1997). Shaping the college curriculum: Academic plans in action. Boston : Allyn \& Bacon.

STEINERT Y. (2000). "Faculty development in the new millennium: Key challenges and future directions". Medical Teacher, vol. 22, $\mathrm{n}^{\circ} 1$, p. 44-50.

SWALES J. (1990). Genre analysis: English in academic and research settings. Cambridge: Cambridge university Press.

TAYLOR K. L. (2005a). "The scholarship of teaching and learning: Building knowledge about learning and teaching in our disciplines ». Communication présentée à la Chaire UNESCO de pédagogie universitaire, Louvainla-Neuve, Belgique.

TAYLOR K. L. (2005b). «Academic development as institutional leadership: An interplay of person, role, strategy and institution". International Journal for Academic Development, vol. 10, $\mathrm{n}^{\circ} 1$, p. 31-46.

TAYLOR K. L. \& REGE COLET N. (2010). « Making the shift from faculty development to educational development: A conceptual framework grounded in practice". In A. Saroyan \& M. Frenay (dir.), Building teaching capacities in higher education: A comprehensive international model. Sterling : Stylus Publishing.

TAYLOR K. L. \& SCHÖNWETTER D. (2002). « Faculty development as institutional leadership: A framework for meeting new challenges ». In A. Goody, J. Herrington \& M. Northcote (dir.), Annual International conference of the Higher education research and development society of Australasia. Perth, Australie : HERDSA, p. 647-654.

WEBB G. (1996). «Theories of staff development: Development and understanding ". International Journal for Academic Development, vol. 1, n 1, p. 63-69.

WEIMER M. \& LENZE L. (1991). « Instructional interventions: A review of the literature on efforts to improve instruction ». In R. Perry \& J. Smart (dir.), Effective teaching in higher education: Research and practice. New York: Agathon Press, p. 205-240.

WILCOX S. (1997). Educational development in higher education. Thèse de doctorat, sciences de l'éducation, University of Toronto, Canada.

WILDMAN T., HABLE M., PRESTON M. \& MAGLIARO S. (2000). "Faculty study groups: Solving "good problems" through study, reflection and collaboration". Innovative Higher Education, vol. 24, n 4, p. 247-263.

WRIGHT W. (1995). Teaching improvement practices: Successful strategies for higher education. Bolton : Anker. 